\title{
An Efficient Deep Learning Model for Olive Diseases Detection
}

\author{
Madallah Alruwaili ${ }^{1}$ \\ Computer Engineering and Networks Department \\ Jouf University \\ Sakaka, KSA \\ Saad Alanazi ${ }^{2}$ \\ Computer Science Department \\ Jouf University \\ Sakaka, KSA
}

\author{
Sameh Abd El-Ghany ${ }^{3}$ \\ Department of Information Systems \\ Mansoura University, Mansoura 35516, Egypt \\ Information Systems Department \\ Jouf University, Sakaka, KSA \\ Abdulaziz Shehab ${ }^{4}$ \\ Department of Information Systems \\ Mansoura University, Mansoura 35516, Egypt \\ Department of Computer Science \\ College of Science and Arts, Jouf University, KSA
}

\begin{abstract}
Worldwide, plant diseases adversely influence both the quality and quantity of crop production. Thus, the early detection of such diseases proves efficient in enhancing the crop quality and reducing the production loss. However, the detection of plant diseases either via the farmers' naked eyes or their traditional tools or even within laboratories is still an error prone and time consuming process. The current paper presents a Deep Learning (DL) model with a view to developing an efficient detector of olive diseases. The proposed model is distinguishable from others in a number of novelties. It utilizes an efficient parameterized transfer learning model, a smart data augmentation with balanced number of images in every category, and it functions in more complex environments with enlarged and enhanced dataset. In contrast to the lately developed state-ofart methods, the results show that our proposed method achieves higher measurements in terms of accuracy, precision, recall, and $F_{1}$-Measure.
\end{abstract}

Keywords-Deep learning; AlexNet; conventional neural networks; plant diseases; olive; feature extraction

\section{INTRODUCTION}

Nowadays and with the advancement of technology such as digital cameras and other new portable devices in image processing, a growing interest has emerged to construct methods that enhance crop production on both quantitative and qualitative bases. A large number of plant diseases contribute to the reduction of the crop production. These diseases mainly influence the state and color of plant leaves, roots, buddings, flowers and fruits. Due to the similar patterns of diseases, it has been proven difficult to point out these minimal differences, rendering their visibility a major challenge. Likewise, inexperienced farmers find it difficult to detect plant diseases with their naked eye. If they were successful, they may not be aware of the appropriate treatment. Thus, the early diagnosis and treatment of these diseases can minimize the losses in the whole crop. To this end, new trends of research have considered the validity of automated methods in detecting plant diseases. The most common techniques for disease detection are machine learning coupled with image processing. These techniques monitor, measure and analyze various images exhibiting common plant diseases. As shown in "Fig. 1", they involve steps that should be taken into consideration for the early identification and diagnosis of plant diseases.

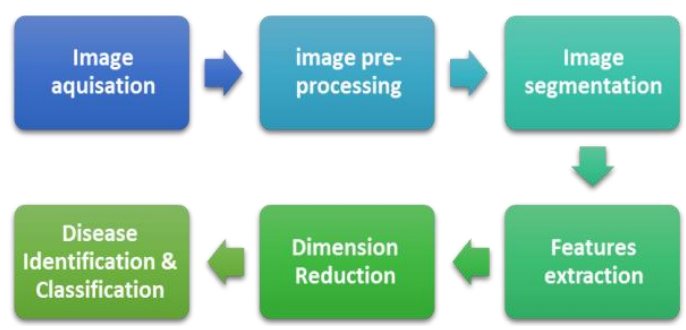

Fig. 1. Basic Steps of Plant Disease Identification and Classification.

Image acquisition aims to obtain and collect images that help in system training. Thus, it is a crucial step given that the accuracy of the system heavily relies on the samples of image for the training purposes [1]. Current studies use either selfcollected image datasets or benchmark datasets such as IPM Images, PlantVillageImages, and APS Image database. (2) As for Image Pre-processing, it seeks to improve the accuracy of disease typology. It includes noise removal, image enhancement techniques, Image quantization, spatial filtering, background removal, resizing and cropping operations. (3) Image Segmentation approach is the third step that can be generally split into two categories: (i) edge detection or (ii) pixel classification. Histogram thresholding, edge detection, Region of Interest (ROI), Otsu's, K-means and Fuzzy c-means are examples for segmentation methods. (4) Feature extraction: in order to identify leaf diseases, we should appeal to appropriate features as a distinguishing descriptor of disease typology. Concerning feature extraction step, it is among the most helpful steps in disease detection and classification as it plays a key role in distinguishing one disease from another. An inappropriate or excessive use of 
features may cause the classification to be over fitting and require long search time. Thus, it is highly recommended to resort to an effective descriptor of the various diseases. The image features can be subsumed under three categories: color, texture, and shape. (5) Dimension Reduction: As a prior step, it is better to pre-process the training data by reducing the dimension of the feature vector so as to maximize the speed and efficiently of the search. Correlation feature selection (CFS) is a central problem to identify the appropriate features for developing a classification model for a particular task via a correlation based method. A good feature subset can enhance the model interpretability, save training time and improve the generalizations by minimizing overfitting. The core assumption is that a good feature set is closely correlated with the class and uncorrelated with other classes. (6) Disease Identification \& classification: a number of automatic methods/models for the detection and classification of plant diseases have been put forward in the recent decades to overcome the limitation of human based visual detection. These methods are based on different machine learning algorithms such as neural network [2], Fuzzy logic, K- nearest neighbor, support vector machine [3], AdaBoost [4], rule base [5], and deep learning [6].

In this paper, we propose an enhanced Convolutional Neural Networks (CNNs) named AlexNet for olive disease detection and classification. Its main contribution is the improvement of the accuracy of olive diseases diagnosis. According to FAO, Olive trees are among the most cultivated plants on the globe and the number of olive trees planted in 2014 is estimated at about 10.2 million hectares. However, olive trees are currently under the threat of a variety of diseases that affect its growth and quality such as canker, Anthracnose, Peacock spot, etc. compared to state-of-art methods, with the aid of an efficient parameterized transfer learning model and a smart data augmentation technique, the results show a predomination although our proposed model functions in more complex environments. The organization of the paper proceeds as follow. Section II provides the relevant literature. An overview of the system framework is presented in Section III. The proposed technique will be laid out in Section IV. In Section V, we will provide a detailed description of the experimentation processes. The results will be discussed in Section VI. Concluding remarks and future work will be given in Section VII.

\section{RELATED WORK}

In response to the costly losses in agricultural production caused by insects and plant diseases, new technological methods have been designed. Computer science is among the disciplines that address these concerns and provide solutions to them. Machine learning, for an instance, plays a key role in detecting such pests and epidemics. In the past decades, a considerable volume of studies with different machine learning algorithm have been executed for Plant disease detection under different environmental conditions, in different countries, and for different plants such as tomato [7], potato [8], rice [9], cassava [10], mango [11], apple [12, 13], general plants [14, 15], and Olive [16, 17], etc. Jagan Mohan et al.[4] presented a system that firstly used SIFT to extract featured from the paddy plant; secondly the AdaBoost classifier was used for disease detection with identification rate $83.33 \%$. After identification, the diseases are recognized with the use of SVM classifier with a recognition rate $91.10 \%$ for SVM and $93.33 \%$ for K-NN. A system that employ fuzzy logic to detect scab disease in apple is presented in [18]. In this study Blob analysis method was used to for feature extraction and the system get accuracy $91.66 \%$ for disease classification. Different deep learning architectures were used in [7] for identifying tomato stresses. The author concluded that Faster RCNN with VGG-16 performed better than other architectures used in the experiments. Monzurul Islam, et al. [8], developed a system to classify the potato diseases. The system firstly mask out the background as well as the green region of the leaves to extract the region that contains disease symptoms. After that two feature extraction methods are used: color and texture, then multiclass SVM classifier is employed for image classification. The accuracy of the system is $95 \%$. In [9], AlexNet deep learning is used to classify rice plant image to three classes, (a) it is infested with golden apple snails; (b) it is afflicted with diseases; and (c) it is normal and healthy; the system provided $91.23 \%$ accuracy. Ramcharan, et al. [10] trained a Tensor flow model of CNN to detect and identify foliar symptoms of diseases in cassava. Thereafter, the trained model was employed in a mobile app. The CNN detection model achieves $94 \pm 5.7 \%$. Singh, et al. [11] presented a multilayer convolutional neural network (MCNN) based approach for identifying Anthracnose fungal disease affect Mango leaves, the system get average accuracy of 97.13\%. Detection of anthracnose lesion in apple fruit using adapted DenseNet model was presented in [12], and it achieved an overall accuracy of $95.57 \%$ for disease identification. Apple leaf diseases using deep-CNNs is proposed in [13], in this system; GoogLeNet Inception structure and Rainbow concatenation (VGG-INCEP model.) are employed to detect five apple leaf diseases (Alternaria leaf spot, Brown spot, Mosaic, Grey spot, and Rust) The classifier achieved a recognition accuracy of $97.14 \%$. AlexNet and GoogLeNet are used in [14] for classification of 54,306 images from PlantVillage to healthy and infected plant leaves. Their model achieved an accuracy of $99.35 \%$. However, their proposal has dramatically collapsed when tested on images taken under different condition. Sladojevic, et al. [15] developed a model using CaffeNet for recognizing 14 different types of plant diseases from healthy leaves in Peach, Apple, and Grapevine. They achieved an average of 96.3\% accuracy in their experimental analysis. Al-Tarawneh, Mokhled [14] worked on olive leaves spot diseases and proposed a novel technique which is a combination of autocropping segmentation and fuzzy c-means clustering. The segmentation part is done by Automatic polygon cropping ROI, while Fuzzy C-means clustering classifier was used to classify the diseases by comparing c- means clustering with kmeans clustering with performance parameters like speed and accuracy. The results show that Fuzzy c-means clustering was found superior than the K-means clustering. A DL model for identification of olive 'quick decline' syndrome called 'abstraction-level fusion' was proposed in [17]. The rate of detection was over $98 \%$, despite the presence of disease in olive fruits. Yet, this system identifies the diseases on the olive leaves only. 


\section{SYSTEM FRAMEWORK}

The present paper aims for a smart system capable of processing olive leaf images with the support of machine learning algorithms and detecting different symptoms of olive diseases. A novel deep learning framework based on AlexNet model was provided to organize the learning process using the transfer learning approach. The proposed model, discussed in more details in the next section, discovers low-level features plant images and in turn detects the disease. The system overview is described as follows: (1) for this study, the data is taken from plant-village dataset. Subsequently, it is enhanced with the olive data collected for Aljouf laboratory. (2) Leaf images are pre-processed via a small window median filter. The filtering process removes noise, and then the images are resized to 256 pixels $\times 256$ pixels. (3) Furthermore, the images are processed with the proposed AlexNet Model. (4) Diagnosis and feedback. "Fig. 2" shows the working steps of the system overview.

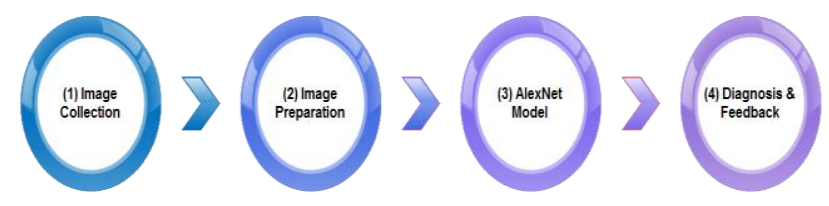

Fig. 2. System Overview.

AlexNet model starts from data collection until up to disease detection process. Occasionally, one of the key steps seeks to efficiently prepare the collected data in a manner fit for the model and assist it in obtaining accurate results. Data preparation includes data augmentation, equalizing the number of images in each class, simple filtering and denoising. Afterwards, the dataset is divided into training set $(80 \%)$ and testing set $(20 \%)$. Optimizing the DL network parameters is accomplished through the training process. The more learned, the more accurate the model becomes in mapping input into its desired output. The trained DL model is then tested against the remaining $20 \%$ unseen images. As a final step, after carrying out a number of experiments and monitoring the output results, the DL network is said to be converged and deployed.

\section{PRoposed MOdEL}

\section{A. AlexNet Description}

Initially, at low-level, a convolutional neural network $(\mathrm{CNN})$ is fed by image's pixel representation defined in PlantVillage dataset. Layers are interconnected together in a
Multi-Layer (ML) architecture. The network is in charge of converting the input visual stimulus into non-local signals. Gradually, the abstraction-level of the signal becomes more complex due to passing through succeeding layers. Lowcomplex features like edges, corners, intensity values, and texture are captured by initial layers while more complex features are formed in a step-by-step format at the higher layers of abstraction. Deep learning (DL) is based on ML techniques where current succeeding layer depends on the output reached from the previous layer. In other words, every two subsequent layers are connected together by neurons. These neurons are nonlinearly transformed layer features. Parameters like weights and biases should be carefully assigned as it determines the accuracy of classification. DL networks are bi-directional neural networks. In the forward direction, image's pixel representation is passed to the input layer which in turn transforms it to next layer. The transformation continues through hidden layers until the output layer. At the output layer, also the decision layer, a decision is made and the residual error is calculated between desired output and the output actually obtained. In the backward direction, the error is fed and backed in order to correct the parameters reversely from output layer to input layer. The model keeps moving forward and backward until achieving satisfied results. Typically, a learning algorithm called back-propagation is utilized for such problems. Stochastic gradient descent (SGD) and its variants, such as mini batch gradient descent [19], ADAM [20], and ADMM [21], have been used to train DNNs. The AlexNet architecture [22] follows the same design pattern as the LeNet-5 [23] architecture. It is a set of stacked convolution layers followed by one or more fully connected layers as shown in "Fig. 3". Optionally, the convolution layers may have normalization and pooling layers. Thereafter, Rectified Linear Unit (ReLu) non-linear activation function is usually used for all the network layers.

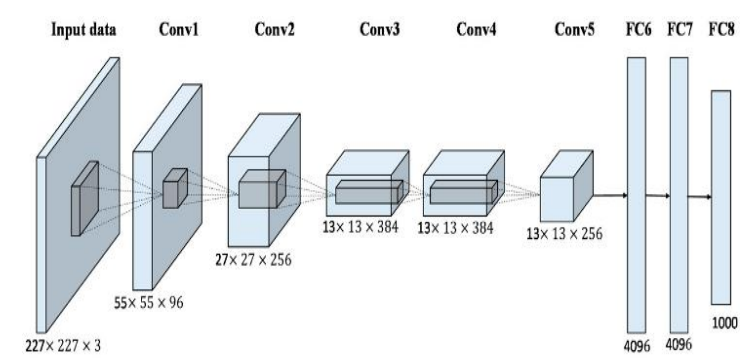

Fig. 3. Classic Structure of AlexNet Deep Neural Network [24].

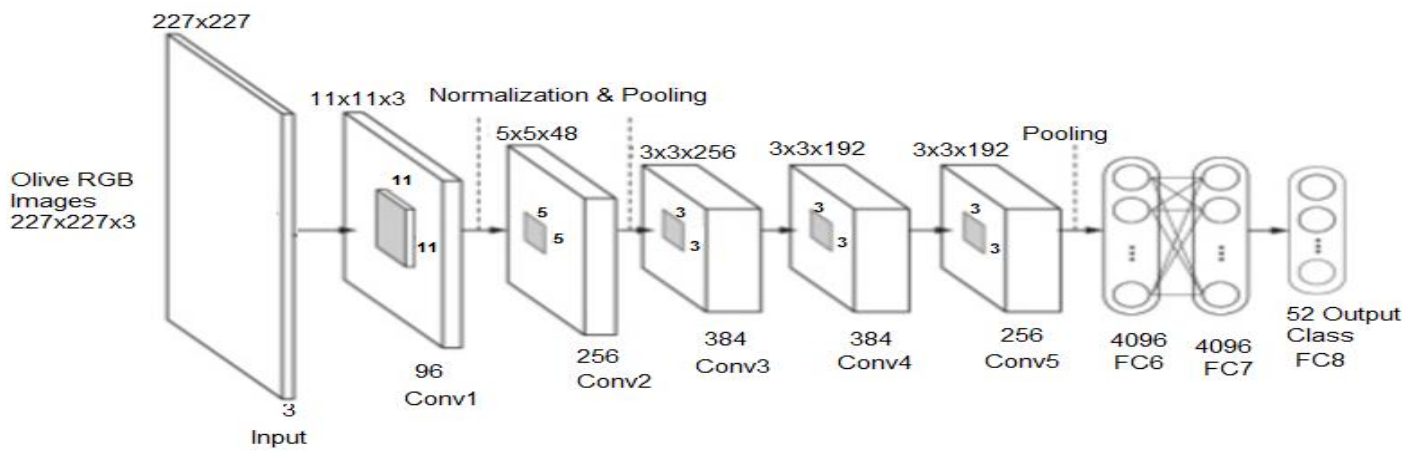

Fig. 4. Structure of the Convolutional Neural Network Employed in this Work. 
As demonstrated in "Fig. 4", our adapted version AlexNet consists of 5 convolution layers (conv1-5), followed by 3 fully connected layers (fc6, fc7, and fc8), and a softMax layer comes at the end. The first two convolution layers (conv1 and conv2) are each followed by both cross channel normalization layer (5 channels per element) and $3 \times 3$ max pooling layer. Meanwhile, the final convolution layer (conv5) is followed by only one pooling layer. The final fully connected layer (fc8) has 52 outputs which equal the total number of classes in our enhanced dataset. Dependently, normalization of fc8 output is exponentially done at the softMax layer. ReLu proceeds for 7 layers of AlexNet from Conv1 through FC7. To reduce the overfitting, both fc6 and fc7 have a dropout ratio of 0.5 overfitting occurs when the model learns massive details about the training data. An overfitting overcome method known as 'sgdm' is used. In addition, novel regularization techniques such as dropout [25] have emerged to reduce overfitting. To build a robust and unbiased model that discovers true parameters, a huge amount of input data with a high changeability must be guaranteed. However, it is an arduous task to obtain a dataset that could cover such changeability in most common realistic applications. To address this issue, as we have not a sufficient large olive dataset, we have to augment the dataset. Data augmentation is accomplished to aid the proposed DL model reaching higher results compared to other methods. Unlike [15], at our Model's training stage, classes suffering a small number of images are geometrically modified with a random transformation such as changing the intensity of RGB channels, flipping, translations or rotations. Moreover, each class has the same number of training images with equal percentage of disease classes.

\section{B. Artificial Data Augmentation}

Some DL models may not be sustainable to natural disorders such as illumination, perspective, and position variability in test images. Ideally, good DL models should overcome both underfitting and overfitting problems. Yet, it is an overambitious goal to achieve in practice. It requires successive trials to reach such a goal. The issue of overfitting occurs when the model learns massive details about the training data. An overfitting overcome method known as 'sgdm' is used. In addition, novel regularization techniques such as dropout [25] have emerged to reduce overfitting. To build a robust and unbiased model that discovers true parameters, a huge amount of input data with a high changeability must be guaranteed. However, it is an arduous task to obtain a dataset that could cover such changeability in most common realistic applications. To address this issue, as we have not a sufficient large olive dataset, we have to augment the dataset. Data augmentation is accomplished to aid the proposed DL model reaching higher results compared to other methods. Unlike [15], at our Model's training stage, classes suffering a small number of images are geometrically modified with a random transformation such as changing the intensity of RGB channels, flipping, translations or rotations. Moreover, each class has the same number of training images with equal percentage of disease classes.

\section{EXPERIMENTATION DETAILS}

\section{A. Dataset Description}

Original PlantVillage dataset has 54,306 images of plant leaves distributed over different 38 classes of 14 crop species and 26 diseases. During the implementation of our experiments, the model is deployed over PlantVillage dataset without any interventions. Nonetheless, due to the absence of olive diseases in it, it was inevitable to enhance the dataset to be further considered. Extra 14 classes of different olive's images are added to PlantVillage dataset and hence 52 classes are formed. Olive Plants within groups were selected from the same olive tree age (25-30 years). To evaluate the presence of a specific disease like Anth, Aspid, Cankers, Frost, Gloe, Hail, etc. in olive trees, sampling is executed according to symptoms in January 2018. "Fig. 5" shows a sample of olive leaf diseases.

\section{B. Performance Metrics}

To evaluate the proposed model, equations (1)-(4) are employed. All of the following metrics are exposed as percentages.

$$
\begin{aligned}
& \text { Accuracy }=\frac{\mathrm{TP}+\mathrm{TN}}{\mathrm{TP}+\mathrm{TN}+\mathrm{FP}+\mathrm{FN}} \\
& \text { Recall }=\frac{\mathrm{TP}}{\mathrm{TP}+\mathrm{FN}} \\
& \text { Precision }=\frac{\mathrm{TP}}{\mathrm{TP}+\mathrm{FP}} \\
& \mathrm{F}_{1}=2 * \frac{\text { Preceision } * \text { Recall }}{\text { Preceision+Recall }}
\end{aligned}
$$

\section{Implementation Details and Parameters}

Due to a controlled collection of PlantVillage dataset, it is expected to obtain highly accurate results. Therefore, we are motivated to further assess the model's performance on an enhanced version of PlantVillage dataset which contains olive images. Such images are scarce (verified 14 class of 120 images). In order to record the olive results, the network is retrained on the new updated version of the dataset. To train the model, parameters summarized in "Table I" are utilized. With the purpose of verification, we perform 10 trials with random sets of $80 \%$ for training data and $20 \%$ for testing data.

TABLE. I. ALEXNet PARAMETERS

\begin{tabular}{|l|l|}
\hline Factor & Value \\
\hline 'WeightLearnRateFactor' & 20 \\
\hline 'BiasLearnRateFactor' & 20 \\
\hline 'InitialLearnRate' & $1 \mathrm{e}-4$ \\
\hline 'MiniBatchSize' & 10 \\
\hline 'MaxEpochs' & 6 \\
\hline Weight decay & 0.0005 \\
\hline Gamma(Y) & 0.1 \\
\hline Batch size & 100 \\
\hline
\end{tabular}




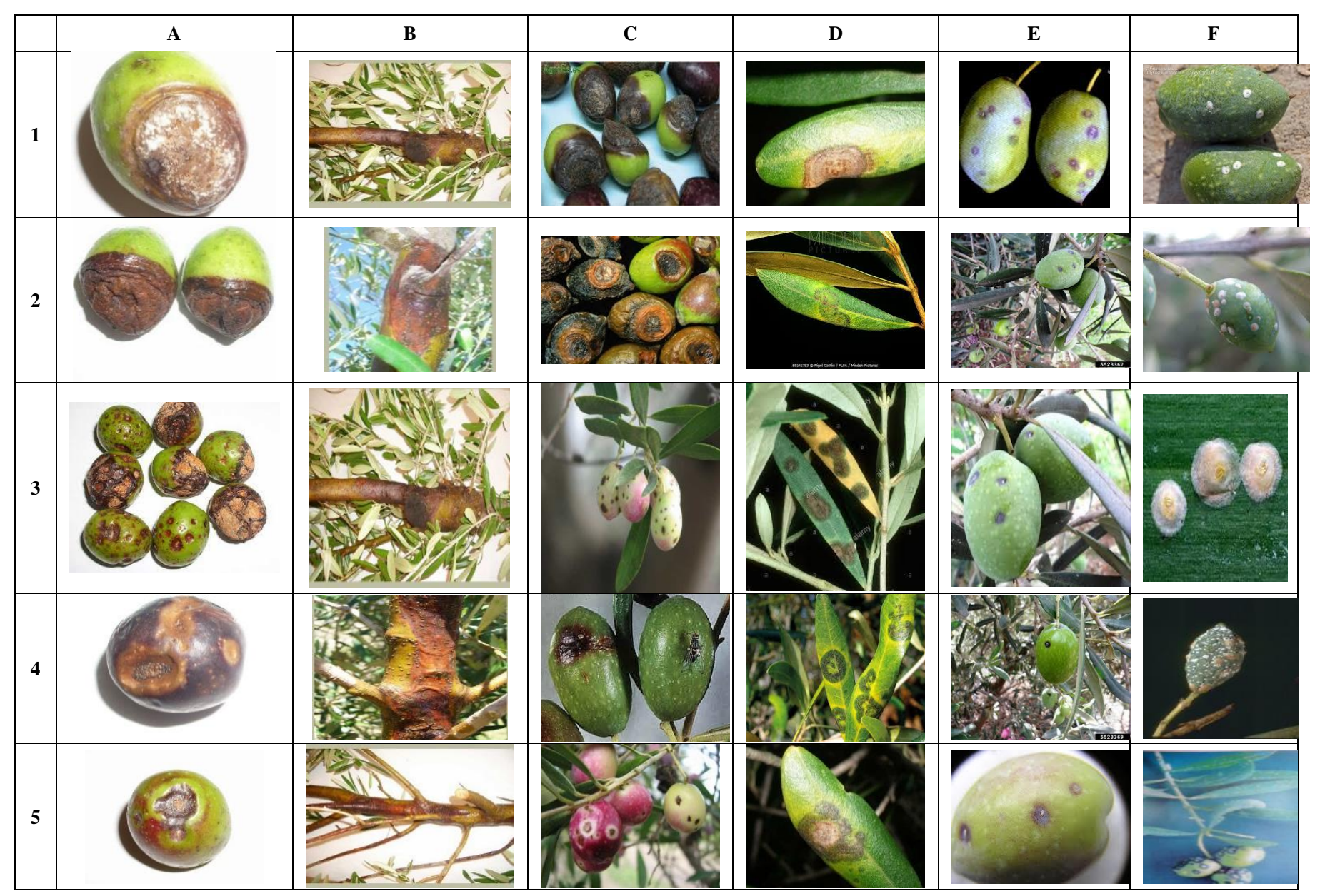

Fig. 5. Sample of Olive Leaf Diseases used in the Experiment. (A) Anthracnose, (B) Canker, (C) Lepra Fruit Rot, (D) Peacock Spot, (E) Parlatoria Oleae, (F) Aspidiotus Nerii.

\section{RESULTS AND DISCUSSION}

Using the proposed DL model network architecture, plant leaves are trained and classified to detect both crop species and disease identity in two main experiments. As mentioned above, one works at the original PlantVillage dataset while the other works at the enhanced dataset using the images acquired from JOUF lab. All experiments are configured to run for a total of 6 epochs each, and they consistently converge after a few steps down in the learning rate. As this paper plans to identify 14 olive diseases, we prefer to document only the results of the final experiment which takes olive Pkant into account. In order to validate the results of the proposed model, the renewed dataset is divided into $80 \%$ training and $20 \%$ testing sets. "Table II" shows the True Positive (TP), False Positive (TP), True Negative (TN), and False Negative (FN) respectively for olive diseases.

Detailed metrics (precision, recall, F1 Measure, sensitivity, and specificity) for each olive disease are depicted in "Table III". The minimum values obtained are highlighted in a red underlined font. 'Peac' has the lowest precision value; 'Frost' has both recall and F1 vlaue, while 'Gloe' has the lowest sensitivity. All specificity measure for all diseases is $100 \%$.

TABLE. II. TP, FP, TN, AND FN FOR OLIVE DISEASES

\begin{tabular}{|l|l|l|l|l|}
\hline Syndrome & TP & FP & TN & FN \\
\hline Anth & 72 & 0 & 2215 & 0 \\
\hline Aspid & 144 & 2 & 2138 & 4 \\
\hline Cankers & 143 & 5 & 2138 & 0 \\
\hline Frost & 1 & 0 & 2283 & 1 \\
\hline Gloe & 419 & 0 & 1867 & 0 \\
\hline Hail & 7 & 1 & 2279 & 5 \\
\hline Lepra & 129 & 2 & 2156 & 0 \\
\hline Macr & 59 & 2 & 2226 & 0 \\
\hline Marciume & 36 & 0 & 2246 & 0 \\
\hline Parl & 6 & 1 & 2280 & 1 \\
\hline Peac & 37 & 4 & 2245 & 3 \\
\hline Phyt & 104 & 0 & 2183 & 1 \\
\hline Pseud & 71 & 1 & 2211 & 3 \\
\hline Tuber & 1 & 0 & 2286 & 0 \\
\hline
\end{tabular}


TABLE. III. OLIVE DISEASES THAT ARE CONSIDERED BY THIS STUDY

\begin{tabular}{|l|l|l|l|l|l|}
\hline Syndrome & Precision & Recall & $\begin{array}{r}\mathbf{F}_{1} \\
\text { Measure }\end{array}$ & Sensitivity & Specificity \\
\hline Anth & 100 & 100 & 100 & 100 & 100 \\
\hline Aspid & 100 & 98.63 & 99.31 & 100 & 100 \\
\hline Cankers & 97.95 & 99.31 & 98.62 & 98 & 100 \\
\hline Frost & 100 & $\underline{50}$ & $\underline{66.67}$ & 99 & 100 \\
\hline Gloe & 100 & 100 & 100 & $\underline{25}$ & 100 \\
\hline Hail & 100 & 100 & 100 & 100 & 100 \\
\hline Lepra & 100 & 100 & 100 & 100 & 100 \\
\hline Macr & 100 & 100 & 100 & 100 & 100 \\
\hline Marciume & 100 & 100 & 100 & 100 & 100 \\
\hline Parl & 100 & 100 & 100 & 88 & 100 \\
\hline Peac & 90.24 & 97.37 & $\underline{93.67}$ & 100 & 100 \\
\hline Phyt & 100 & 100 & 100 & 97 & 100 \\
\hline Pseud & 100 & 100 & 100 & 100 & 100 \\
\hline Tuber & 100 & 98.63 & 99.31 & 100 & 100 \\
\hline
\end{tabular}

"Fig. 6" alongside "Table IV" summarizes the remarked results in contrast to a number of the state-of-art methods. The proposed method achieves an overall accuracy about $99.11 \%$ which is the highest mark. Besides, it has $99.49 \%, 99.11 \%$, and $99.29 \%$ in terms of precision, recall, and F1 measure respectively. These metrics are the highest as opposed to other methods. It has been noticed that our proposed model outperforms all other methods in terms of overall accuracy, precision, recall, and F1 measure. This singularity in measurements is a result of efficient artificial augmentation, balanced number of class images, optimal parameters assignment, smart network configuration, and usefulness of transfer learning. The problems arising in most traditional attempts [25-28] to detect plant diseases follow from handengineered features, image enhancement techniques, and labor-intensive methodologies. These traditional attempts are lean to either a small number images in a class or a limited variety of classes of crops. Unlike [17], the proposed model AlexNet model is trained over an enhanced version of PlantVillage dataset. Thus, it is more general for multiple disease identification for apple, tomato, and olive leaf diseases. Moreover, it relies on augmentation and achieved an overall accuracy of $99.11 \%$ for disease identification.

To conclude, it has been observed that the performance of DL models in image classification has made a remarkable progress in the past few years [29-31]. Previous traditional approaches such as SIFT [32], HoG [33], SURF [34], etc., and the likes were based on hand-engineered features extraction methods. These approaches heavily depend on predefined features. They also lack the transfer learning. In other words, they fail once the problem at hand is renewed or major changes are introduced to the dataset.

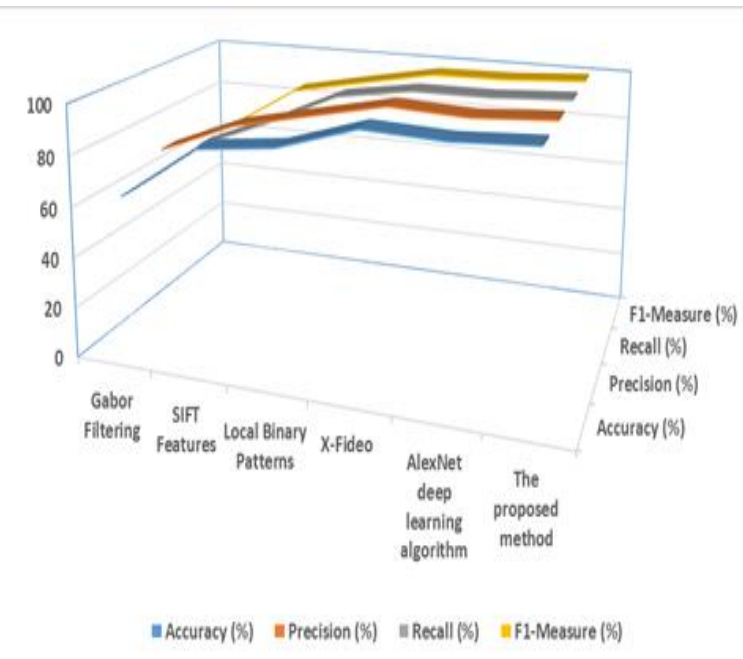

Fig. 6. The Proposed Method Compared to State-of-Art Methods.

TABLE. IV. Proposed Method COMPARED to STATE-OF-ART Methods

\begin{tabular}{|l|l|l|l|l|}
\hline The proposed method & Accuracy $(\%)$ & Precision (\%) & Recall (\%) & F -Measure (\%) \\
\hline $\begin{array}{l}\text { Background Suppressing Gabor Energy Filtering } \\
\text { [26] with RBF-SVM [27]. }\end{array}$ & $63.11 \pm 11.91$ & $72.44 \pm 14.30$ & $65.28 \pm 21.74$ & $65.52 \pm 15.15$ \\
\hline SIFT Features[28] and RBF-SVM [27]. & $84.91 \pm 17.44$ & $85.19 \pm 20.07$ & $77.87 \pm 43.61$ & $84.65 \pm 18.13$ \\
\hline $\begin{array}{l}\text { Uniform Local Binary Patterns [35] and RBF-SVM } \\
\text { [27]. }\end{array}$ & $88.55 \pm 16.71$ & $92.12 \pm 17.68$ & $92.24 \pm 6.16$ & $90.95 \pm 11.97$ \\
\hline X-Fideo (LeNet deep learning algorithm) [17]. & $98.60 \pm 1.47$ & $98.82 \pm 2.63$ & $97.18 \pm 2.71$ & $96.89 \pm 3.45$ \\
\hline AlexNet deep learning algorithm [15]. & $97.38 \pm 1.89$ & $97.42 \pm 1.33$ & $97.37 \pm 1.45$ & $97.36 \pm 2.45$ \\
\hline The proposed method & $99.11 \pm 0.75$ & $99.49 \pm 0.83$ & $99.11 \pm 1.29$ & $99.29 \pm 1.63$ \\
\hline
\end{tabular}




\section{CONCLUSION AND FUTURE WORK}

Early detection of plant diseases has been taken as a positive move to maintain and enhance crop quality and reduce production loss to the minimum. As a result, DL approaches gain wide acceptance worldwide due to their accuracy and efficiency in plant disease detection field. The remarked results compared to a number of state-of-the-art methods are promising. Our proposed method achieves an overall accuracy of $99.11 \%$ which is the highest mark. Besides, it has $99.49 \%, 99.11 \%$, and $99.29 \%$ in terms of precision, recall, and $F_{1}$ measure respectively. It outperforms all other methods when it comes to overall accuracy, precision, recall, and $F_{1}$ measure. More interestingly, although the model training consumes ample time, the classification during testing runs quickly in a few seconds even on a CPU. Therefore, the model could be easily implemented on a smartphone. In the future, a smartphone-assisted crop disease diagnosis will be targeted, and the proposed model will be available at Mobil apps.

\section{ACKNOWLEDGMENT}

We are indebted to the Deanship of Scientific Research at Jouf University for funding this work through General Research Project under grant number (39/766). We also extend our gratitude to Olive Research Center as well as Camel and Range Research Center for their support during our data collection of olive diseases and pests.

\section{REFERENCES}

[1] Kaur, S., S. Pandey, and S. Goel, Plants disease identification and classification through leaf images: A survey. Archives of Computational Methods in Engineering, 2019. 26(2): p. 507-530.

[2] ABDUllaKASIM, W., et al., An images analysis technique for recognition of brown leaf spot disease in cassava. Tarım Makinaları Bilimi Dergisi, 2011. 7(2): p. 165-169.

[3] Bhange, M. and H. Hingoliwala, Smart farming: Pomegranate disease detection using image processing. Procedia Computer Science, 2015. 58: p. 280-288.

[4] Mohan, K.J., M. Balasubramanian, and S. Palanivel, Detection and recognition of diseases from paddy plant leaf images. International Journal of Computer Applications, 2016. 144(12).

[5] Phadikar, S., J. Sil, and A.K. Das, Rice diseases classification using feature selection and rule generation techniques. Computers and electronics in agriculture, 2013. 90: p. 76-85.

[6] Amara, J., B. Bouaziz, and A. Algergawy. A Deep Learning-based Approach for Banana Leaf Diseases Classification. in BTW (Workshops). 2017.

[7] Fuentes, A., et al., A robust deep-learning-based detector for real-time tomato plant diseases and pests recognition. Sensors, 2017. 17(9): p. 2022.

[8] Islam, M., et al. Detection of potato diseases using image segmentation and multiclass support vector machine. in 2017 IEEE 30th canadian conference on electrical and computer engineering (CCECE). 2017. IEEE.

[9] Atole, R.R. and D. Park, A multiclass deep convolutional neural network classifier for detection of common rice plant anomalies. International Journal of Advanced Computer Science and Applications, 2018. 9(1): p. 67-70.

[10] Ramcharan, A., et al., A mobile-based deep learning model for cassava disease diagnosis. Frontiers in Plant Science, 2019. 10: p. 272.

[11] Singh, U.P., et al., Multilayer Convolution Neural Network for the Classification of Mango Leaves Infected by Anthracnose Disease. IEEE Access, 2019. 7: p. 43721-43729.
[12] Tian, Y., et al., Detection of Apple Lesions in Orchards Based on Deep Learning Methods of CycleGAN and YOLOV3-Dense. Journal of Sensors, 2019. 2019.

[13] Jiang, P., et al., Real-Time Detection of Apple Leaf Diseases Using Deep Learning Approach Based on Improved Convolutional Neural Networks. IEEE Access, 2019. 7: p. 59069-59080.

[14] Al-Tarawneh, M.S., An empirical investigation of olive leave spot disease using auto-cropping segmentation and fuzzy C-means classification. World Applied Sciences Journal, 2013. 23(9): p. 12071211.

[15] Prasanna Mohanty, S., D. Hughes, and M. Salathe, Using Deep Learning for Image-Based Plant Disease Detection. arXiv preprint arXiv:1604.03169, 2016.

[16] Sladojevic, S., et al., Deep neural networks based recognition of plant diseases by leaf image classification. Computational intelligence and neuroscience, 2016. 2016.

[17] Cruz, A.C., et al., X-FIDO: An effective application for detecting olive quick decline syndrome with deep learning and data fusion. Frontiers in plant science, 2017. 8: p. 1741.

[18] Kour, V.P. and S. Arora, Fruit Disease Detection Using Rule-Based Classification, in Smart Innovations in Communication and Computational Sciences. 2019, Springer. p. 295-312.

[19] Goodfellow, I., Y. Bengio, and A. Courville, Deep learning. 2016: MIT press.

[20] Kingma, D.P. and J. Ba, Adam: A method for stochastic optimization. arXiv preprint arXiv:1412.6980, 2014.

[21] Taylor, G., et al. Training neural networks without gradients: A scalable admm approach. in International conference on machine learning. 2016.

[22] Krizhevsky, A., I. Sutskever, and G.E. Hinton. Imagenet classification with deep convolutional neural networks. in Advances in neural information processing systems. 2012.

[23] LeCun, Y., et al., Backpropagation applied to handwritten zip code recognition. Neural computation, 1989. 1(4): p. 541-551.

[24] Han, X., et al., Pre-trained alexnet architecture with pyramid pooling and supervision for high spatial resolution remote sensing image scene classification. Remote Sensing, 2017. 9(8): p. 848.

[25] Srivastava, N., et al., Dropout: a simple way to prevent neural networks from overfitting. The Journal of Machine Learning Research, 2014. 15(1): p. 1929-1958.

[26] Cruz, A.C., B. Bhanu, and N.S. Thakoor, Background suppressing Gabor energy filtering. Pattern Recognition Letters, 2015. 52: p. 40-47.

[27] Chang, C.-C., " LIBSVM: a library for support vector machines," ACM Transactions on Intelligent Systems and Technology, 2: 27: 1--27: 27, 2011. http://www. csie. ntu. edu. tw/ cjlin/libsvm, 2011. 2.

[28] Liu, C., J. Yuen, and A. Torralba, Sift flow: Dense correspondence across scenes and its applications. IEEE transactions on pattern analysis and machine intelligence, 2011. 33(5): p. 978-994.

[29] Simonyan, K. and A. Zisserman, Very deep convolutional networks for large-scale image recognition. arXiv preprint arXiv:1409.1556, 2014.

[30] Zeiler, M.D. and R. Fergus, Visualizing and understanding convolutional networks (2013). arXiv preprint arXiv:1311.2901, 2013.

[31] Szegedy, C., et al. Inception-v4, inception-resnet and the impact of residual connections on learning. in Thirty-First AAAI Conference on Artificial Intelligence. 2017.

[32] Lowe, D.G., Distinctive image features from scale-invariant keypoints. International journal of computer vision, 2004. 60(2): p. 91-110.

[33] Dalal, N. and B. Triggs. Histograms of oriented gradients for human detection. in international Conference on computer vision \& Pattern Recognition (CVPR'05). 2005. IEEE Computer Society.

[34] Bay, H., et al., Speeded-up robust features (SURF). Computer vision and image understanding, 2008. 110(3): p. 346-359.

[35] Almaev, T.R. and M.F. Valstar. Local gabor binary patterns from three orthogonal planes for automatic facial expression recognition. in 2013 Humaine Association Conference on. 